\title{
OR-64
}

\section{Chemical Constituents of Mitrella Kentii (Annonaceae)}

\author{
Ainnul Hamidah Syahadah Azizan ${ }^{*}$ and A. Hamid A. Hadi \\ Department of Chemistry, Faculty of Science, University Of Malaya $50603 \quad$ Kuala Lumpur; \\ E-mail: ainnul_azizan@yahoo.com
}

Mitrella kentii which belongs to the Annonaceae family is a tree-climbing liana found in the Malaysian Peninsula, Islands of Sumatra, Borneo, and New Guinea. This species was studied in 1972 for their alkaloids, 1997 and 2012 for their non-alkaloid constituents. In this study, chalcones, Desmosdumotin C (1) and their tautomer, 2-cinnamoyl-3-hydroxy-5-methoxy-4,6,6-trimethylcyclohexa-2,4dienone (2), flavanone, 7-hydroxy-5,6-dimethoxy-2-phenylchroman-4-one (3), oxoaporphine alkaloids, Liriodenine (4) and Atherospermidine (5) and also terpenoids, 3-oxostigmasterol (6) and 3,25-dioxostigmasterol (7) have been isolated from this species . All these compounds were isolated for the first time from Mitrella kentii except Liriodenine (4). The isolated compounds were elucidated using spectroscopic techniques such as UV, IR,1D and 2D NMR and mass spectroscopy and by comparison their spectral data with those previously reported in the literatures. Desmosdumotin C (1) showed the anti-ulcer activity.

Keywords: Annonaceae, Mitrella kentii, Alkaloid, Desmosdumotin C, Anti- ulcer activity. 